\title{
MEASUREMENTS ON SLAC LINAC RF SYSTEM FOR LCLS OPERATION*
}

\author{
R. Akre, P. Emma, P. Krejcik, SLAC, Stanford, CA 94309, USA
}

\section{Abstract}

The Linac Coherent Light Source (LCLS) project [1] at SLAC uses a dense $15 \mathrm{GeV}$ electron beam passing through a long undulator to generate extremely bright $\mathrm{x}$ rays at 1.5 angstroms. The project requires electron bunches with a nominal peak current of $3.5 \mathrm{kA}$ and bunch lengths of $0.020 \mathrm{~mm}$ (70fs). The RF stability required by the bunch compressors is tighter than what is currently required to run experiments. Measurements to determine how well the existing linac will meet the new requirements are ongoing. Presented is an update on the measurements and how they pertain to LCLS.

\section{LINAC PHASE MEASUREMENTS FOR LCLS RF REQUIREMENTS}

Results of two different types of phase measurements done on the SLAC Linac to evaluate phase stability as it pertains to LCLS operation are presented. The specifications for LCLS are given in Table 1. Of the five LCLS linacs listed only L2 and L3 contain a significant number of klystrons distributed over large distances. L0, L1, and LX are located within 50 meters of the injector for LCLS. LCLS klystron allocation is shown in figure 1.

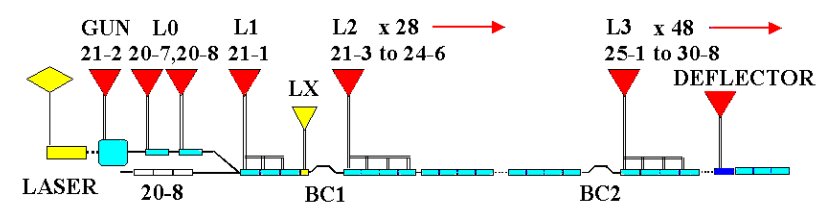

Figure 1 : LCLS Klystron Allocation

The phase tolerances in Table 1 are the total phase jitter in each LCLS linac. For the multi-klystron linacs, individual klystrons are allowed higher jitter as long as the linac total RF phase jitter as seen by the beam if within the tolerance.

Table 1: LCLS RF stability requirements.

\begin{tabular}{|l|c|c|c|}
\hline Linac & Klystrons & Phase rms & Amp. \% rms \\
\hline L0 & 2 & $0.1^{\circ} \mathrm{S}$ & 0.1 \\
\hline L1 & 1 & $0.1^{\circ} \mathrm{S}$ & 0.1 \\
\hline LX & 1 & $0.30^{\circ} \mathrm{X}$ & 0.25 \\
\hline L2 & 28 & $0.07^{\circ} \mathrm{S}$ & 0.07 \\
\hline L3 & 48 & $0.07^{\circ} \mathrm{S}$ & 0.05 \\
\hline
\end{tabular}

The RF reference system, in order to meet LCLS requirements, must start out with a low phase noise oscillator. The low noise RF must then be distributed amplified and measured with enough accuracy to meet LCLS requirements. To meet the above requirements integrated system noise levels of $0.03^{\circ} \mathrm{rms}$ S-band are desired.
The first set of measurements show the phase noise of the $476 \mathrm{MHz}$ low level RF distribution system used to run the 2 mile, 30 Sector, linac. Measurements are done at the existing linac sector 0 master oscillator, at sector 21 , and at sector 30. LCLS L2 and L3 will use sectors 21 to 30 of the SLAC linac.

Data on the phase stability of klystrons within sectors was taken and analyzed. Correlated and uncorrelated noise components were then calculated.

\section{PHASE NOISE MEASUREMENTS}

\subsection{Phase Noise Measurement System}

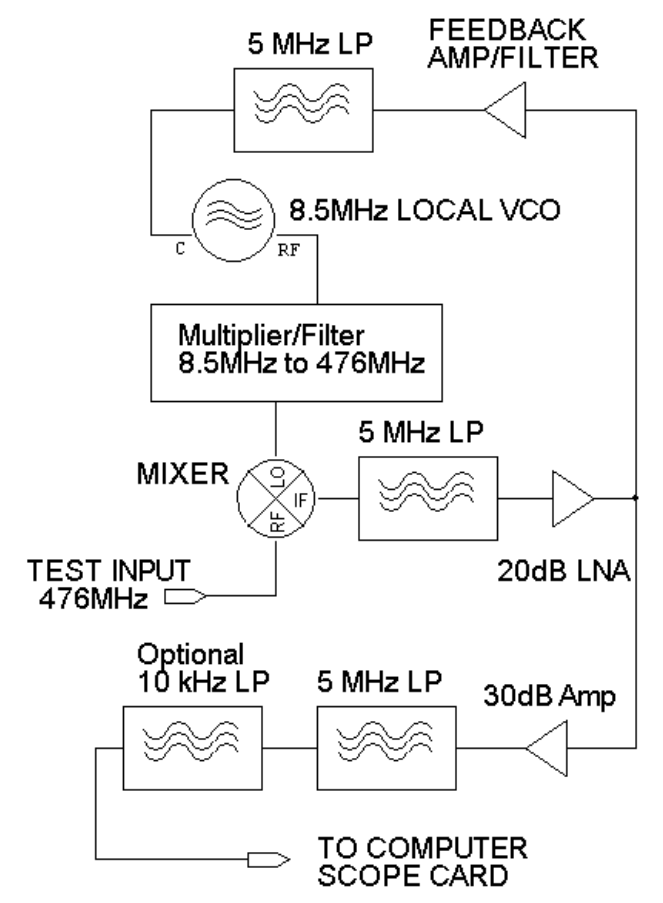

Figure 2: Diagram - Phase Noise Measurement System.

The system in figure 2 was used to measure the phase noise in the phase reference system. The system uses a low phase noise $8.5 \mathrm{MHz}$ oscillator multiplied to $476 \mathrm{MHz}$. A mixer is used to measure the difference in phase between the low noise oscillator and the Test Input. The phase error signal is then amplified and split. One side is used for feedback to lock the oscillator to the Test Input signal with a $1 \mathrm{~Hz}$ bandwidth. The other side is amplified and input into a $20 \mathrm{MHz}$ digitizing scope card in a P.C. The phase noise at the Test Input can be measured if it is

*Supported by the U.S. Department of Energy, contract DE-AC0376SF00515: PAC2001 TPAH105: SLAC-PUB-8820 
above that of the local oscillator and beyond the bandwidth of the phase lock loop. The inherent noise floor of the measuring system is shown for comparison in figures 3 and 4 .

\subsection{Data}

Two measurements were done at each of 3 locations, sector 0 master oscillator, sector 21 , and sector 30 . In the first measurement the phase signal was digitized at $40 \mathrm{kHz}$ with a $10 \mathrm{kHz}$ anti-aliasing filter. This measurement gives phase noise data from about $4 \mathrm{~Hz}$ to $10 \mathrm{kHz}$. The data for the master oscillator in Sector 0 is shown in figure 3. The noise level of the measurement system is also shown as a reference.

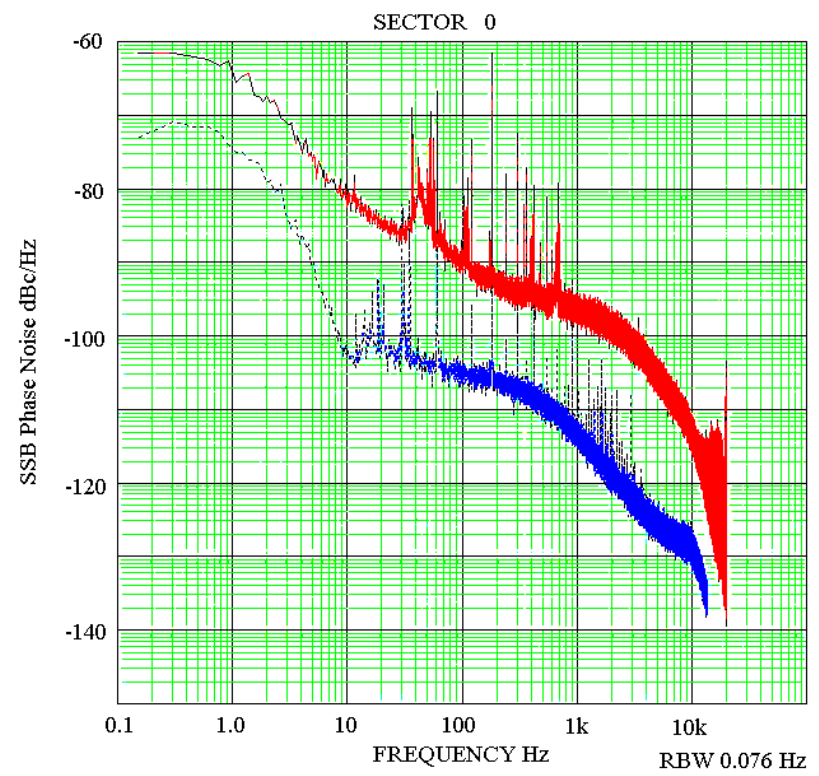

Figure 3: Low Freq. Phase Noise Sector 0 and Test Unit

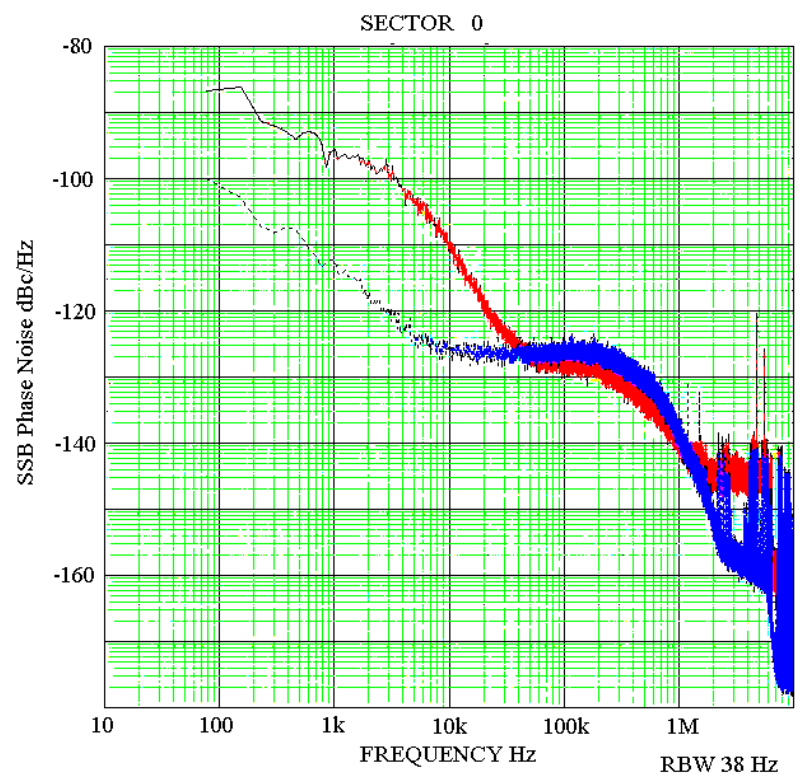

Figure 4: High Freq. Phase Noise Sector 0 and Test Unit

The second measurement, figure 4 for Sector 0 , is digitized at $20 \mathrm{MHz}$ with a $5 \mathrm{MHz}$ anti-aliasing filter. The figure shows noise level of the measurement system to be higher than the phase noise of the Test Input for frequencies between $40 \mathrm{kHz}$ and $20 \mathrm{MHz}$. For these frequencies the $-30 \mathrm{~dB}$ per decade between 10 and $20 \mathrm{kHz}$ could be extrapolated to the noise floor of $-145 \mathrm{dBc} / \mathrm{Hz}$.

The data taken at sector 20 and 30 looked within error to be the same as sector 0 .

\subsection{Comments on Data}

The LCLS beam passes through accelerator structures that integrate the RF for $830 \bullet \mathrm{S}$. Although the fill time reduces high frequency noise as seen by the beam, the RF phase measurement system has a bandwidth of $10 \mathrm{MHz}$. The phase noise of the system up to $10 \mathrm{MHz}$ will affect the accuracy of the measurement.

Phase measurements are done with respect to a reference. If the phase noise is low enough in frequency, the path difference between the reference and measured point of the noise will be a small fraction of a wavelength. For example, an RF source has a $1 \mathrm{kHz}$ phase noise vector of $-40 \mathrm{dBc}$ and $R F$ is split, one side for a reference and the other side through a drive system and to a monitor point. If the difference in path length between the reference and monitor point is $1 \mu \mathrm{S}$, the $1 \mathrm{kHz}$ noise vector would have moved $.3^{\circ}$ or 0.005 radians. The measurement system would see this $-40 \mathrm{dBc}$ noise signal as a-40+20log(.005) or $-86 \mathrm{dBc}$. If there were no difference in path length the phase of the reference and the monitor point would be the same and the system would not detect phase noise. The reference RF and drive RF are split at the start of each sector. The major contributor to the path difference between the reference and the beam is the SLAC Linac Energy Doubler, SLED, cavity at the output of each klystron. The cavity has a $3 \bullet \mathrm{S}$ fill time. This will set a lower frequency limit on the phase noise, which affects the klystron phase measurement, at about $100 \mathrm{kHz}$.

The noise floor of the existing oscillator, $-145 \mathrm{dBc} / \mathrm{Hz}$, will give a noise level of $0.086 \cdot \mathrm{rms}$ at $2856 \mathrm{MHz}$ when integrated over $10 \mathrm{MHz}$ as follows:

$-145 \mathrm{dBc} / \mathrm{Hz} \times 10 \mathrm{MHz}=-75 \mathrm{dBc}$ SSB integrated noise.

Total noise $=-75 \mathrm{dBc} \times 2$ sidebands $=-72 \mathrm{dBc}$

$-72 \mathrm{dBc}$ noise power $=10^{-3.6}$ radians $\mathrm{rms}$ at $476 \mathrm{MHz}$

$10^{-3.6}$ radians rms at $476 \mathrm{MHz}=0.086 \cdot \mathrm{rms}$ at $2856 \mathrm{MHz}$

The above noise levels are not sufficient for LCLS operation. The desired noise levels for the LCLS phase distribution and measurement system would be a factor of 4 below the LCLS specification for RF phase stability. A lower noise oscillator such as one similar to the one used in the measurement system will reduce noise levels to a level at which LCLS stability can be accurately measured.

\section{KLYSTRON STABILITY MEASUREMENTS}

The stability of the high powered klystrons and modulators at SLAC is easily measured using the control system. On March 28, 2001 measurements were taken at a time just after the sun came up. During this time the phases in the SLAC linac RF distribution system are changing rapidly with outside temperature rise. The BPM 
Buffered Data Acquisition system of the SLAC Control System allows 512 consecutive points of phase measurements from each of the klystrons to be taken. Nine data sets were recorded for 114 klystrons in 15 sectors at 10 and $30 \mathrm{~Hz}$.

\subsection{Data and Analysis}

Data from set number 5 is shown in table 2. The data set, 512 points in 51.2 seconds, was taken at $07: 33$ on March 28, 2001. Between 07:00 and 08:00 the outside temperature rose $4 \cdot \mathrm{F}$. The table shows the standard deviation, STD, of the 512 data points for each klystron in each sector. An entry of zero means the klystron was not running at the time, data was not collected. The first row is the sector number, below which is the STD of the 512 points of data for each of the eight klystrons in the sector. The row labeled "std $\Sigma$ " is the STD of the summed data as in equation 1 .

$$
\operatorname{std} \sum=\frac{\operatorname{STD}}{8}\left(\text { FOR I }=1 . .512\left(\sum_{\text {KLYS }-1}^{8} \text { DATA }_{\mathrm{I}}\right)\right)
$$

This is the rms phase jitter as seen by the measurement system.

Table 2: Klystron Phase Standard Deviations (Degrees SBand) Set 5.

$\begin{array}{rrrrrrrrr}\text { Sect } & 2 & 3 & 4 & 5 & 6 & 9 & 11 & 12 \\ \text { Kly. } & & & & & & & & \\ 1 & 0.150 & 0.319 & 0.128 & 0.158 & 0.065 & 0 & 0.135 & 0.094 \\ 2 & 0.115 & 0.085 & 0.122 & 0.101 & 0.097 & 0 & 0.332 & 0.109 \\ 3 & 0 & 0.079 & 0.169 & 0.072 & 0.178 & 0.097 & 0 & 0.102 \\ 4 & 0.118 & 0.070 & 0.194 & 0.069 & 0.095 & 0.097 & 0.086 & 0.100 \\ 5 & 0.080 & 0.107 & 0.154 & 0.091 & 0.082 & 0.102 & 0.097 & 0.079 \\ 6 & 0.088 & 0.074 & 0.185 & 0.189 & 0.150 & 0.091 & 0.075 & 0.834 \\ 7 & 0.110 & 0.088 & 0.839 & 0.150 & 0.072 & 0.120 & 0.204 & 0.099 \\ 8 & 0.104 & 0.093 & 0.171 & 0.151 & 0.095 & 0.083 & 0.072 & 0.147 \\ \text { std } \sum & 0.062 & 0.062 & 0.158 & 0.080 & 0.055 & 0.065 & 0.070 & 0.126 \\ \sqrt{\Sigma V} & 0.042 & 0.049 & 0.118 & 0.046 & 0.039 & 0.040 & 0.064 & 0.110 \\ \text { Corr } & 0.045 & 0.039 & 0.105 & 0.065 & 0.038 & 0.051 & 0.030 & 0.061\end{array}$

$\begin{array}{rrrrrrrr}\text { Sect } & 13 & 14 & 15 & 16 & 17 & 18 & 19 \\ \text { Kly } & & & & & & & \\ 1 & 0.068 & 0.145 & 0.104 & 0.085 & 0.247 & 0.111 & 0.067 \\ 2 & 0.110 & 0.111 & 0.085 & 0.151 & 0.250 & 0.117 & 0.078 \\ 3 & 0.094 & 0.065 & 0.100 & 0.099 & 0.270 & 0.102 & 0.074 \\ 4 & 0.098 & 0.068 & 0.098 & 0.091 & 0.251 & 0.118 & 0.119 \\ 5 & 0.202 & 0.149 & 0.090 & 0.099 & 0.228 & 0.203 & 0.109 \\ 6 & 0 & 0.107 & 0.119 & 0.129 & 0.219 & 0.111 & 0.116 \\ 7 & 0.103 & 0.273 & 0.116 & 0.120 & 0.212 & 0.086 & 0 \\ 8 & 0.078 & 0.248 & 0.101 & 0.147 & 0.253 & 0.105 & 0.045 \\ \text { std } \sum & 0.056 & 0.073 & 0.052 & 0.081 & 0.219 & 0.080 & 0.057 \\ \sqrt{ } \sum \mathrm{V} & 0.043 & 0.058 & 0.036 & 0.042 & 0.086 & 0.044 & 0.034 \\ \text { Corr } & 0.035 & 0.045 & 0.037 & 0.069 & 0.202 & 0.067 & 0.045\end{array}$

The next row, $\sqrt{ } \sum \mathrm{V}$, is one-eighth the square root of the sum of the squares of the STD of the eight klystrons above. If the data were uncorrelated normal distributions this would be the expected STD for the sector. This is the uncorrelated portion of the sector STD given in the row above.

The last row, Corr, is the square root of the difference of the square of the above two rows. This is the correlated part of the sector STD.

If all klystron data is summed to get the STD for all 114 klystrons the result is $0.0422^{\circ} \mathrm{S}$. The square root of the sector variances is $0.0252^{\circ} \mathrm{S}$. This is the uncorrelated part of the above data. The correlated part of STD for all klystrons is $0.0339^{\circ} \mathrm{S}$.

The other 8 sets of data are quite similar to the above set 5 .

\subsection{Comments on Data and Analysis}

For both klystrons in a sector and sectors in the linac the correlated and uncorrelated parts of the STD are about equal. The average STD of the 114 klystrons is $0.14^{\circ} \mathrm{S}$. For a sector the average STD is $0.086^{\circ} \mathrm{S}$. Using this number the STD, or rms phase jitter, for L2 of LCLS is expected to be $0.068^{\circ} \mathrm{S}$ over 51 seconds. This assumes a sum of four sectors with $0.086^{\circ} \mathrm{S}, 50 \%$ of which is correlated and $50 \%$ of which is uncorrelated. Although the rms phase jitter of $0.068^{\circ} \mathrm{S}$ is within LCLS requirements we must be able to do better in order to insure reliability of the system.

In this data set klystrons 3-1, 4-7, 11-2, 11-7, 12-6, 13$5,14-7,14-8,18-5$ and sector 17 are above $0.20^{\circ} \mathrm{S}$ rms jitter. Although with current PEP-II linac operation this level of phase jitter is acceptable. This level of stability is not acceptable for LCLS operation.

For single klystron L0 and L1 almost half the klystrons would meet the requirements. Currently SLAC selects high stability klystrons and support electronics for critical locations. For L0 and L1 a high stability system would easily meet the requirements.

\section{MEETING LCLS RF PHASE REQUIREMENTS}

The stability of the existing system is close to meeting the requirements. A higher level of regular maintenance will be required for LCLS operation. The measurements presented here show stability for up to 52 seconds. Phase drifts in the reference phase distribution to the klystrons still require evaluation and beam-based feedback systems need to be developed. Some work has been done[1] but more is required to determine if the existing RF distribution system will meet LCLS requirements.

\section{REFERENCES}

[1] LCLS Design Study Report, SLAC-R-521 (1998)

[2] R. Akre, V. Bharadwaj, P. Emma, P. Krejcik, "SLAC Linac RF Performance for LCLS", SLAC-PUB-8574, and THC11, LINAC 2000, Monterey, CA, Aug. 2000 